OPEN

SUBJECT AREAS:

IMAGING

NEUROSCIENCE

NEUROANATOMY

NEURODEVELOPMENTAL

DISORDERS

Received

18 May 2011

Accepted

4 July 2011

Published

25 July 2011

Correspondence and requests for materials should be addressed to G.M.R. Igianmichele. ratto@sns.it)

*S.L. and E.P. contributed equally to the study.

\section{The short-time structural plasticity of} dendritic spines is altered in a model of Rett syndrome

\author{
Silvia Landi ${ }^{1}, 2 *$, Elena Putignano ${ }^{1,3 *}$, Elena Maria Boggio ${ }^{3}$, Maurizio Giustetto ${ }^{4}$, Tommaso Pizzorusso 3,5 \\ \& Gian Michele Ratto $1,2,3$
}

${ }^{1}$ NEST, Scuola Normale Superiore, Pisa, Italy, ${ }^{2}$ NEST, Institute Nanoscience CNR, Pisa, Italy, ${ }^{3}$ Institute of Neuroscience CNR, Pisa, Italy, ${ }^{4}$ National Institute of Neuroscience-ltaly and Department of Anatomy, Pharmacology and Forensic Medicine, University of Turin, Italy, ${ }^{5}$ Dipartimento di Psicologia, Università di Firenze- Florence, Italy.

The maturation of excitatory transmission comes about through a developmental period in which dendritic spines are highly motile and their number, form and size are rapidly changing. Surprisingly, although these processes are crucial for the formation of cortical circuitry, little is known about possible alterations of these processes in brain disease. By means of acute in vivo 2-photon imaging we show that the dynamic properties of dendritic spines of layer V cortical neurons are deeply affected in a mouse model of Rett syndrome (RTT) at a time around P25 when the neuronal phenotype of the disease is still mild. Then, we show that $24 \mathrm{~h}$ after a subcutaneous injection of IGF-1 spine dynamics is restored. Our study demonstrates that spine dynamics in RTT mice is severely impaired early during development and suggest that treatments for RTT should be started very early in order to reestablish a normal period of spine plasticity.

ett syndrome (RTT) is a neurodevelopmental disorder characterized by a period of apparently normal development of 6-18 months followed by regression and onset of a variety of symptoms including motor abnormalities, mental retardation, epilepsy and anxiety ${ }^{1}$. Most cases of RTT involve mutations of methylCpG-binding protein 2 (MeCP2), a gene encoding a methylated DNA-binding protein that regulates gene transcription, mRNA splicing and chromatin structure ${ }^{2}$. However, how these genetic defects translate into RTT symptoms is still unknown. It has been proposed that synaptic alterations constitute a main substrate of the disease symptoms $s^{3,4}$, but only subtle alterations of neuronal density, dendritic arborisation and number of dendritic spines (the main site of excitatory synapses) were found in both patients $\mathrm{s}^{5,6}$ and mouse models in which $\mathrm{MeCP} 2$ has been deleted ${ }^{7}$. So far, the available studies ${ }^{7}$ have been performed in fixed tissue thus not allowing the visualization of the dynamic processes underlying juvenile dendritic spine maturation. These involve the formation and removal of highly motile filopodia that can be stabilized and transformed into more stable mature spines. These processes can occur in a time scale of minutes and they are altered in response to manipulations that do not lead to manifest variations of spine density, suggesting that the short-time dynamic regulation of spine structure plays an important role in shaping connectivity of neural circuits ${ }^{8}$. To fill this gap, we analyzed dendritic spine dynamics in somatosensory cortex by 2-photon time lapse imaging in MeCP2 null mice crossed with Thy-GFP line $e^{9,10}$. At the onset of the disease (3-4 weeks of age ${ }^{11,12}$ ) we found deep alterations in the dynamics of dendritic spines and filopodia. Later on at P40 when the maturation of the connectivity in the somatosensory cortex is complete, the dynamics of dendritic spines in RTT mice was identical to control, although a reduction in spine density was observed in mutants. Finally, we found that $24 \mathrm{hrs}$ after a single subcutaneous injection of IGF-1, a molecule known to pass the blood brain barrier $\left(\mathrm{BBB}^{13}\right)$ and of proposed therapeutic potential in RTT ${ }^{14-16}$, spine motility was completely restored in the cerebral cortex of RTT mice.

\section{Results}

We crossed the MeCP2 null-mutants ${ }^{9}$ with a mouse line expressing green fluorescent protein (GFP) in a sparse subset of layer $\mathrm{V}$ pyramidal neurons (GFP-M line; 10). Initially we analyzed juvenile (P25-26) GFP-MeCP2-KO mice (RTT mice), at a time that precedes the onset of evident RTT neurological symptoms ${ }^{11,12}$. We found that the general architecture of layer $\mathrm{V}$ pyramids was normal but the density of dendritic spines and filopodia was reduced in mutant mice. Also the length of spines was slightly but significantly shorter when compared with controls 

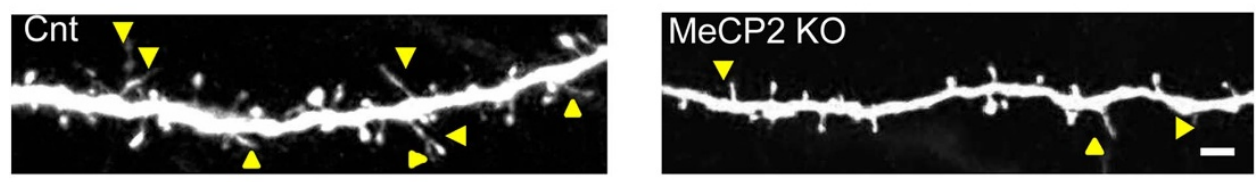

\section{B Fixed tissue}
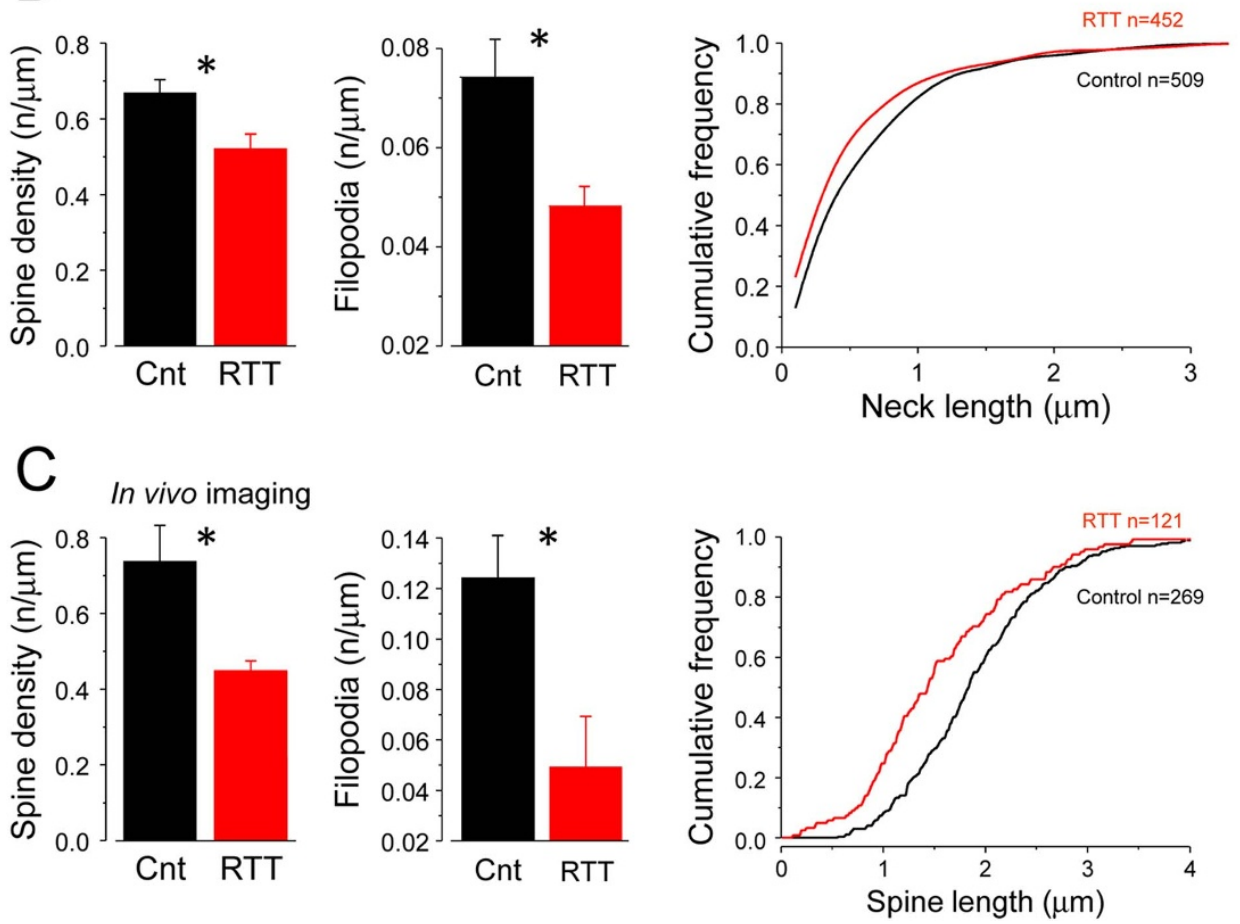

Figure $1 \mid$ Morphological alterations of dendritic spines in GFP-MeCP2-KO mice. (A) Confocal micrographs showing GFP-positive dendrites of layer V pyramidal neurons in the S1 cortex of perfused-fixed control (cnt) and GFP-MeCP2-KO mice (RTT). Filopodia-like spines are indicated with arrowheads. Scale bar $2.5 \mu \mathrm{m}$. B, C, left and center panels) Both spines and filopodia are less dense in the RTT mice ( $\mathrm{t}$-test. B: spines, $\mathrm{p}<0.03$; filopodia, $\mathrm{p}<0.05$; $\mathrm{C}$ spines, $\mathrm{p}=0.01$; filopodia, $\mathrm{p}=0.02$ ) when evaluated in fixed tissue (B) or in vivo (C). $\mathrm{B}, \mathrm{C}$ right panels) Cumulative distributions of spine necklength (B, fixed tissue) and of spine length (C, in vivo) revealed a general decrement of spine length in the RTT mice (KS-test, B: $p<0.01$; $C$ : $p<0.001)$. Column plots show the mean and the standard error of the mean.

(Fig. 1). These data emerge both from images obtained in fixed tissue (Fig. 1B) and in vivo (Fig. 1C), thus indicating that the GFP-MeCP2$\mathrm{KO}$ mice show a reduction of the density of dendritic spines, as previously reported for the $\mathrm{MeCP} 2-\mathrm{KO}$ mice $^{7}$ and that the conditions of in vivo imaging do not alter the basic morphometric characteristics of dendritic spines. Moreover, these data confirms that synaptic connectivity in the cortex of RTT mice might be already impaired at this early developmental stage ${ }^{7}$. Then, we proceeded to measure the short time dynamics of dendritic spines in the RTT mice by means of 2-photon imaging through a cranial window. After identification of a suitable dendrite, we acquired $\mathrm{z}$-stacks of the selected fields at $5 \mathrm{~min}$ interval over a period of 50-60 min (Fig. 2A). Time lapse imaging revealed a dramatic effect of the $\mathrm{MeCP} 2$ deletion on the short time structural plasticity of spines (see also Supplementary Video V1 and V2 online). In RTT mice motility of dendritic spines (expressed as variation of length) was severely reduced (Fig. 2B). The observed reduction was largely due to a decreased fraction of filopodia and to their great stability compared to control. In the subset of spines with a clearly defined head, we measured its volume in each temporal frame, and the distribution of the fluctuations of the head volume for both control and RTT mice are shown in Fig. 2C. It is clear that the spine head volume is far more stable in the cortex of MeCP2 mutants compared with control mice. In summary, these data demonstrate that both filopodia and more mature spines are affected by MeCP2 deletion. Fluctuations of length and volume report two different functional properties of dendritic spines, being the length correlated with connectivity and with the spine-dendrite coupling, while the head volume is correlated to the size of the postsynaptic density and with synaptic strength ${ }^{17-20}$. These parameters are uncorrelated in our sample, as also shown in previous work on normal mice (Fig. 2D;20). During normal cortical development, filopodia almost disappear, spines reach their mature form and structural stability increases: thus we expected that the differences in motility observed at P25 would fade away later on as the circuitry stabilizes. Indeed, time lapse imaging performed in $\mathrm{P} 40$ mice showed that at this age spine motility in control mice is decreased to levels comparable to the MeCP2 null mice (Fig. 3), whereas spine density remains significantly impaired in $\mathrm{MeCP} 2$ mutants. Thus, the deletion of MeCP2 impairs spine motility during the critical period for cortical plasticity and synaptogenesis. The impact of MeCP2 loss of function on early development suggests that any treatment directed at attenuating RTT condition should be performed during the critical period for cortical plasticity. IGF-1 is required for the maintenance of dendritic spines in the adult cerebellum ${ }^{21}$, and it has been shown to improve the RTT symptoms in the MeCP2 null mice ${ }^{16}$. We wondered whether IGF-1 might also act early during postnatal development on the short-term dynamics of dendritic spines. Mice have been treated with a single injection of the long form of IGF-1 


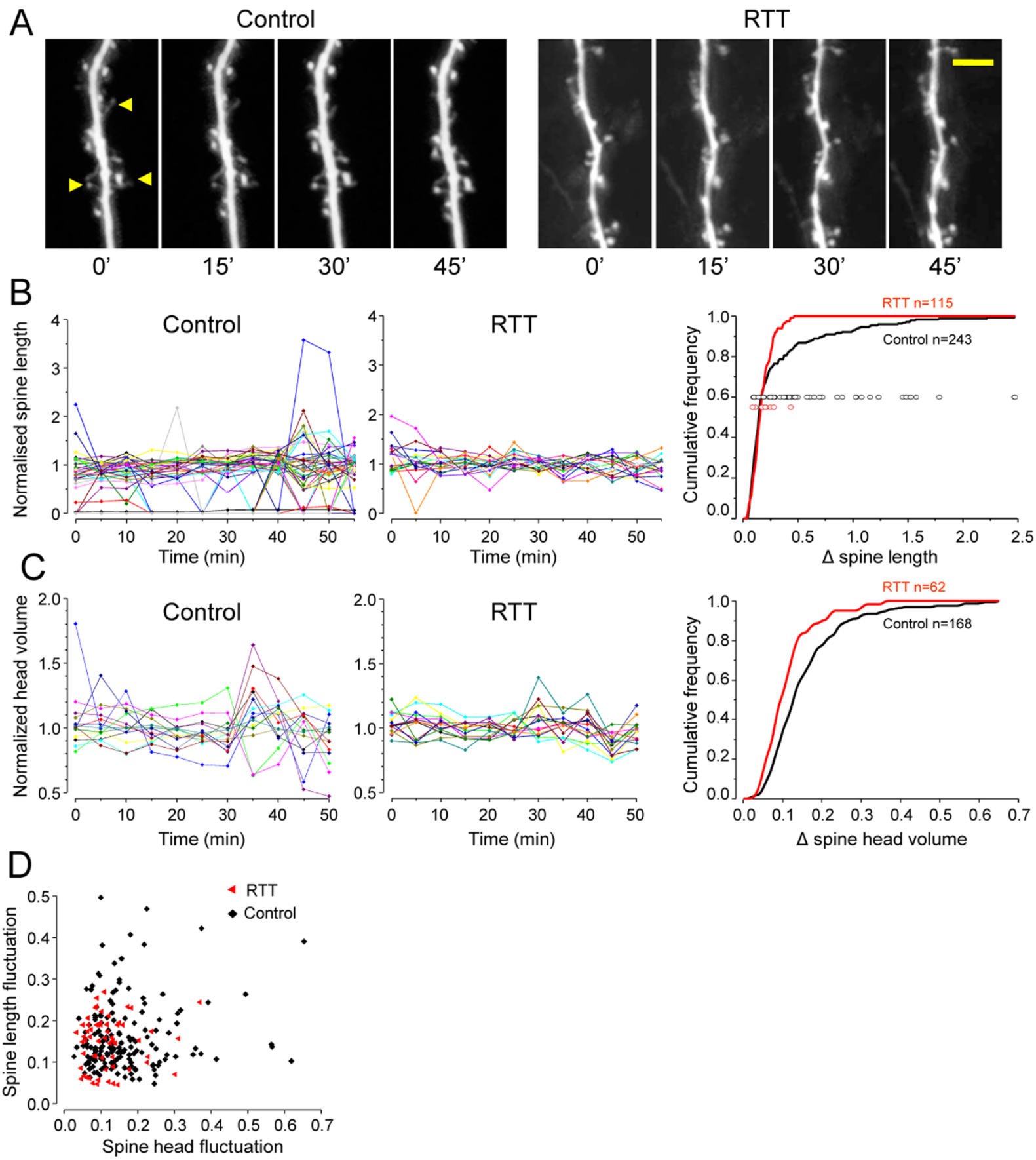

Figure $2 \mid$ Dendritic spine dynamics is altered in a model of Rett syndrome at P25-26. (A) Time lapse imaging in a control and a RTT mouse at P25. Arrowheads points to motile spines and filopodia. Scale bar $5 \mu \mathrm{m}$. B, left) Representative data showing the time course of spine length in a control and a RTT mouse. Right) Cumulative distribution of the average motility showing that in the RTT mice dendritic spines are more stable (cnt, N=7; RTT, N=5; KS-test, $\mathrm{p}=0.014$ ). The dots represent the average motility of each filopodia. $\mathrm{C}$, left) Time course of the fluctuations of the spine head volume in a control and in a RTT mouse. Right) The cumulative distribution of the average fluctuation of the spine head volume shows that in the RTT mice the spine head is more stable (KS-test, $\mathrm{p}=0.002$ ). (D) Scatter graph showing that there is no correlation between spine motility and fluctuation of spine head volume in RTT and control mice.

$24 \mathrm{~h}$ before two-photon imaging (Fig. 4A; 22). The morphometric analysis of these data evidenced a recovery in spine length and no effects on spine density after IGF-1 treatment (Fig. 4B). Furthermore, we observed a complete recovery in dendritic spine dynamics tested both by the fluctuation of spine length and by the fluctuation of the volume of the spine head (Fig. 4C, and Supplementary Video V3 online). Although filopodia density showed a small but not significant increment, their stability was drastically modified by IGF-1 treatment. Stability of each filopodia has been quantified by computing a coefficient defined as the number of frames in which each filopodia is visible divided by the total sequence length: thus a coefficient of 1 means that the filopodia is present during the entire imaging session. The distribution of filopodia coefficients of stability (Fig. 4D) shows that in the RTT mice $90 \%$ of filopodia are present during the entire imaging period whereas in controls this percentage is only $40 \%$. The treatment with IGF- 1 strongly increased filopodia turnover in the RTT mice to $60 \%$, control becoming similar to that of mice. Interestingly, the IGF-1 treatment did not cause 
A

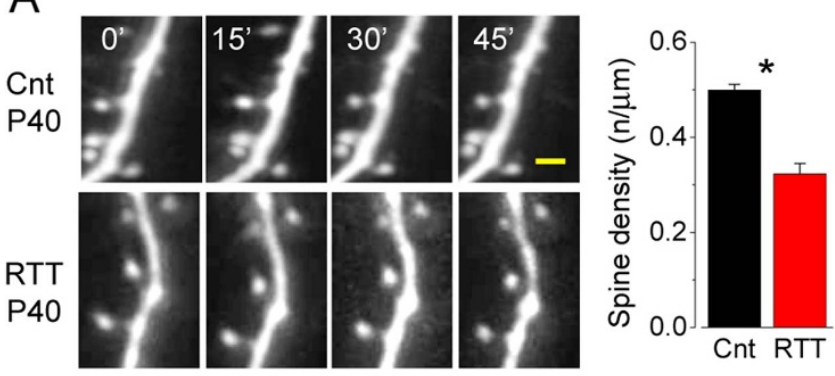

B

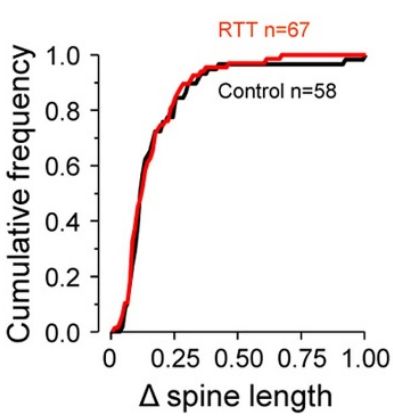

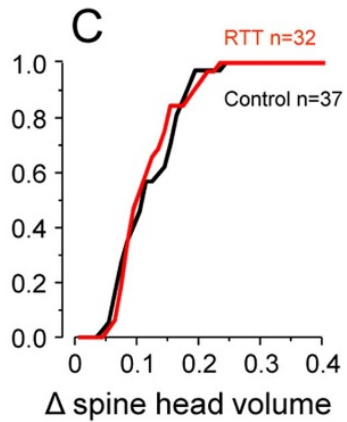

$\Delta$ spine head volume

Figure $3 \mid$ No differences in spine dynamics in older mice ( $>$ P40). (A left) Time lapse imaging in a control and a RTT mouse at P40. Scale bar $2.5 \mu \mathrm{m}$. A right) Spine density is significantly reduced at $\mathrm{P} 40$ between control and RTT mice ( $\mathrm{cnt}, \mathrm{N}=5, \mathrm{KO}, \mathrm{N}=4$; t-test, $\mathrm{p}<0.001$ ) (B) Cumulative distributions of spine length fluctuations revealed no differences between control and RTT at P40 (KS, p $=0.733)$.

(C) Cumulative distributions of spine head volume fluctuations showed no differences between control and RTT at P40 (KS, p=0.676).

any change in spine motility in control wild type mice, possibly indicating that IGF-1 availability is not the rate limiting factor of the processes controlling structural plasticity during normal development (Fig. 4C).

\section{Discussion}

Our experiments have shown that the dynamic properties of dendritic spines in cortical pyramidal neurons are deeply affected by MeCP2 mutations suggesting that synaptic defects in RTT are even more complex than what was previously indicated by neuroanatomical studies. Indeed, an anomalous cortical circuitry is established in the cortex of RTT mice even before the full appearance of the neurophysiological expression of RTT. The impairment of the cellular mechanisms regulating short-term dynamics of dendritic spines and filopodia occurs early during the postnatal development, in a critical window for cortical plasticity and synaptogenesis. Thus, the alterations in spine development should prevent the normal formation and maturation of synaptic connectivity. MeCP2 lossof-function mutation has also been associated with several other neurodevelopmental disorders, i.e. epilepsy, Angelman syndrome, autism, infantile encephalopathy, juvenile onset of schizophrenia, Parkinsonism and X-linked mental retardation in both male and females ${ }^{1}$. Therefore, impairments in spine short-time structural plasticity could represent a common pathogenic trait in several brain disease. IGF-1 is a growth factor important for brain development and plasticity ${ }^{13}$ widely expressed in the CNS during normal development ${ }^{23}$ that strongly promotes neuronal cell survival, synaptic maturation $^{23,24}$, and functional plasticity in the developing cortex ${ }^{25}$. The putative relationship between RTT and IGF-1 is highlighted by the observations that IGF-1 concentration is lower in the cerebrospinal fluid of autistic children ${ }^{26}$ and that $\mathrm{MeCP} 2$ regulates directly the expression of the insulin-like growth factor binding protein 3, thus regulating IGF-1 action ${ }^{27}$. Indeed, its pharmacological potential is

enhanced by the ability to pass the $\operatorname{BBB}(26,13)$, property not shared by other trophic factors, such as $\mathrm{BDNF}^{28}$. IGF-1 is capable of crossing the $\mathrm{BBB}$, both in the tri-peptide form ${ }^{1-3}$ IGF- $1^{29}$ and in the long form $^{30-34}$. Notably, the BBB architecture is well suited to transport serum IGF-1 into the brain, although the details of this process are still incomplete. Both brain vessels, choroid plexus epithelium and perivascular glial end-feet express IGF-1 receptors ${ }^{35,36}$, thus IGF-1 is transferred by endothelial cells into the perivascular space surrounded by glial end-feet able to endocytose IGF- $1^{37}$. Finally, IGF-1 could be transferred to adjacent neurons through trans-endocytosis or a related process. In conclusion, the established vision is that IGF-1 can move into the brain through two ways: serum IGF-1 passes into the CSF by physiological oscillations in blood IGF-1 levels ${ }^{32}$ in a tonic modality even if, serum IGF-1 levels usually remain very steady. A second proposed mechanism involves the regulated passage of serum IGF-1 through an activity-dependent mechanism ${ }^{13}$.

Here, we have shown that IGF-1 acts on dendritic spines early in development, during the period of enhanced structural plasticity. Interestingly, the effects of IGF-1 treatment were present already one day after injection. At this time, we observed no recovery of spine density, but the increased turnover of filopodia suggests that as the treatment progresses, more spines could be added to the dendrites ${ }^{16}$. The cellular details of IGF-1 action are not completely clarified and the mechanisms altered in the MeCP2-KO mouse and that are rescued by IGF-1 are still unknown. We can envision two different lines of actions, that may, to some extent, coexist. 1) IGF-1 acts on synaptic transmission by converging on the signalling pathways of PI3K/ pAkt/PSD-95 ${ }^{38}$ and MAPK $^{39}$, similarly to BDNF. Indeed, both pAkt signalling ${ }^{40}$ and BDNF expression are impaired in MeCP2 null mice $^{41}$ and different mutations in MeCP2 correlates with different expression levels of BDNF and with a specific severity of RTT in general $^{42,41}$. Thus, exogenous IGF-1 might act by vicariating the reduced signalling of BDNF. Specifically, IGF-1 has been shown to elevate excitatory postsynaptic currents significantly ${ }^{43}$ : the action of IGF-1 on excitatory transmission, is strongly implied by the fact that treating with IGF-1 neurons cultured from patients carrying different MeCP2 mutations, resulted in an increase in glutamatergic synapse number, suggesting that the drug treatment could correct defective excitatory transmission ${ }^{44}$.

2) It is also possible that IGF-1 might act more directly on spine dynamics, by contributing to the regulation of actin. In muscle cells it has been shown that IGF-1 modulates the activity of N-WASP resulting in enhanced actin filament formation ${ }^{45}$. Since, N-WASP is also a crucial regulator of actin during spine formation and plasticity ${ }^{46}$ the possibility arises that actin based motility might be directly influenced by IGF-1 availability. In summary, our study has shown that structural plasticity is impaired in the MeCP2 mouse, at a time in which cortical circuitry is refined by experience. Then we have shown that the pharmacological treatment with IGF-1 can recover most of the deficit of structural plasticity. Our results indicate that time lapse imaging of the short-time dynamics of dendritic spines can be used as a powerful screening tool for designing therapeutic strategies targeting pathologies where structural plasticity is impaired. Finally, our data suggest that treatments should be administered early on during postnatal development, when cortical plasticity is still enhanced.

\section{Methods}

Preparation of the GFP-MeCP2+/- mice. All experimental procedures were carried out in compliance with the institutional guidelines of the Italian Ministry for Public Health. The GFP-MeCP2-KO mice were derived from heterozygous B6.129SF1-MeCP2 $2^{\text {tmlJae }}$ knock-out females (MeCP2 $\left.+/-\right)^{9}$. Females were originally crossed to C57BL6 for one generation, followed by breeding amongst offspring of the same generation with breeder changes, and were maintained on a mixed background to reduce mortality and to obtain the high numbers of mice required by the experiments. Age matched littermates were used in all experiments to control for possible effects of genetic background unrelated to the MeCP2 mutation ${ }^{47}$. $\mathrm{MeCP} 2+/-$ females were then crossed with Thy-1 GFP transgenic mice (line $\mathrm{M}^{10}$ ) and only mice expressing GFP at cortical level were used for this study. GFP control 
A

P0

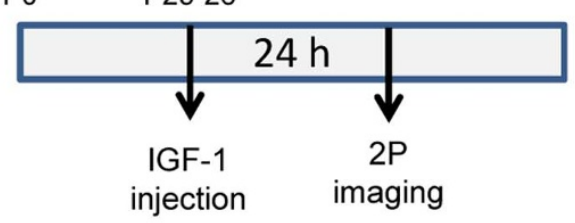

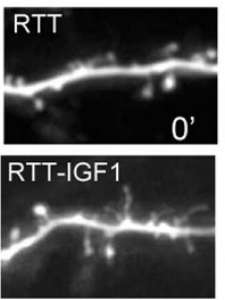

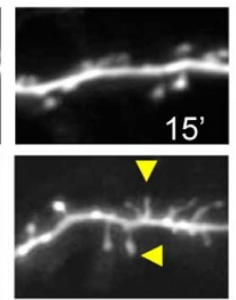

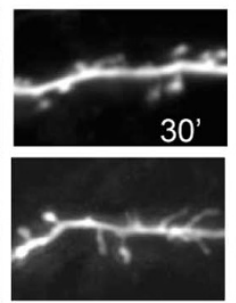
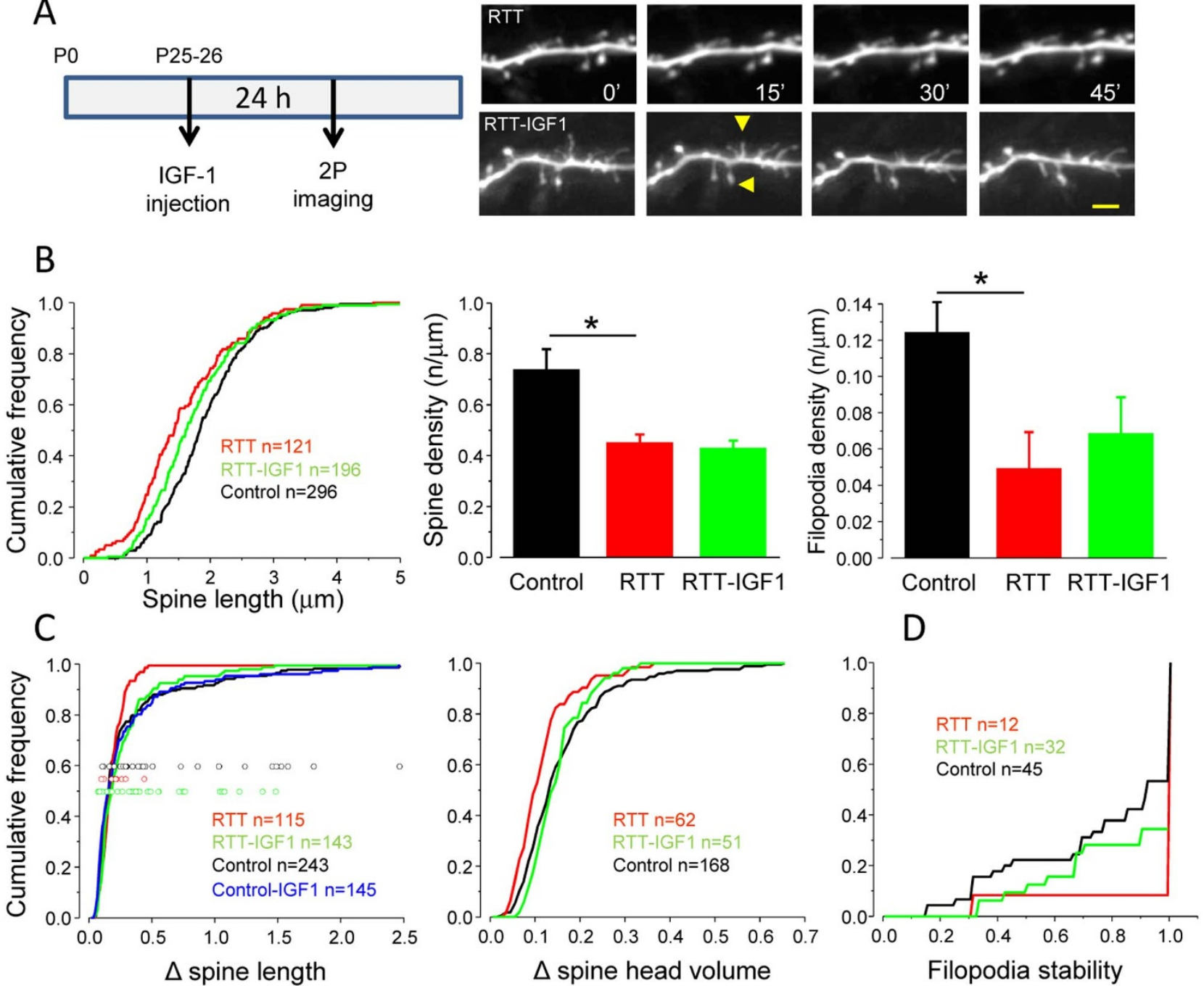

Figure $4 \mid$ IGF-1 treatment restores spine dynamics in RTT mice. (A left) Protocol of the experiment. Right) Time lapse imaging in a RTT mouse and in a RTT mouse treated with IGF-1. Scale bar $2 \mu \mathrm{m}$. B left) Cumulative distribution of spine length showed that the treatment with IGF-1 lead to a partial recovery of the spine length in RTT mice (data replotted from fig. 1 for control and RTT mice for comparison; KS-test, cnt $v s$ RTT-IGF-1, p=0.004; RTT $v s$ RTT-IGF-1, $\mathrm{p}=0.031$ ). B center and right) Both spine density and filopodia densities were not affected by IGF-1 (One-Way ANOVA, post-hoc Tukey test, $\mathrm{p}<0.05)$. C left) Data showing the distributions of the fluctuation of spine length in the specified conditions. In the RTT mice dendritic spines are more stable than controls (replotted from fig. 2), while spine motility becomes similar to controls after IGF-1 treatment (cnt $v s$ RTT-IGF-1, KS test, $\mathrm{p}=0.170$; RTT $v$ RTT-IGF-1, $\mathrm{p}=0.010$ ). The dots represent the average motility of each filopodia. IGF-1 treatment per se does not cause any effect in control mice (cnt $v s$ cnt IGF-1, $\mathrm{p}=0.16$ ); C,center) Distribution of the fluctuations of the spine head volume in the specified conditions. The data show that after IGF-1 administration RTT spine head fluctuations become normal (KS-test: cnt $v s$ RTT-IGF1, p=0.50; RTT $v s$ RTT-IGF1, $\mathrm{p}=0.006$ ). (D) Cumulative distribution showing that while filopodia in RTT mice are very stable in comparison to filopodia in controls, $24 \mathrm{~h}$ after IGF-1 treatment they become less stable, with parameters intermediate between untreated RTTs and controls (KS-test; cnt $v s$ RTT, $\mathrm{p}=0.029$; $\mathrm{cnt} v s$ RTT-IGF1, $\mathrm{p}=0.469$; RTT $v s$ RTT-IGF1, $\mathrm{p}=0.528)$.

mice were separated from GFP-MeCP2-KO mice by PCR screening ${ }^{12}$. We employed only mice with an expression level of GFP high enough to provide a sufficient quality of the imaging.

Confocal microscopy on fixed tissue. GFP-MeCP2 null mice $(\mathrm{N}=4)$ and GFP control littermates $(\mathrm{N}=4)$ were anesthetized with an intraperitoneal injection of chloral hydrate and perfused with $4 \%$ paraformaldehyde in $0.1 \mathrm{M}$ phosphate buffer (PB). Brains were post-fixed overnight in the same fixative solution, washed several times in $0.1 \mathrm{M} \mathrm{PB}$ and then cut coronally into $90-\mu \mathrm{m}$ sections on a vibratome (Leica VT 1000S, Germany). Immunolabelling on free-floating sections with an antibody against GFP ( $1: 5000$, rabbit, Synaptic Systems) was used to enhance GFP fluorescence signal. Briefly, sections were blocked for 1 hour at room temperature (RT) using a solution of $3 \%$ bovine serum albumin, $0.05 \%$ Triton X-100 and $10 \%$ normal goat serum (NGS) in phosphate buffer saline (PBS) and then incubated overnight at RT with anti-GFP antibody in a PBS solution containing $3 \%$ NGS and $0.05 \%$ Triton X-100. Sections were then washed in PBS and subsequently incubated with a fluorescent secondary antibody (Alexa488 goat anti-rabbit; $1: 1000$ ) in a solution of $3 \%$ NGS, $0.05 \%$ Triton X-100 in PBS for 1 hour at RT, rinsed several times in PBS and finally mounted on glass slides with Dako fluorescent mounting medium. Sections were viewed with a confocal microscope (Zeiss LSM-5 Pascal, Germany) equipped with a $40 \times$ oil-immersion objective (pinhole: 1.0 Airy unit). At least 10 z-stack images consisting of $10-15$ sections $(512 \times 512$ pixels $)$ spaced $0.5 \mu \mathrm{m}$ apart were acquired in the layer II-III of the S1 cortex of each animal from distal tertiary branches (dendritic segment of length in the 35 to $60 \mu \mathrm{m}$ range) of layer V pyramidal neurons as in 48 . Dendritic segments and spines were analyzed quantitatively using ImageJ (NIH, USA, public domain) by an observer blind to the experimental conditions. Measure of spine density was performed on projected $\mathrm{z}$-stack images and spine number was divided by the length of the dendritic segment to compute the density expressed as number of spines per micrometer. If dendritic spines were too packed to clearly separate them from each other, we turned to serial stack images to delineate individual spines. The measurement of spine neck length was performed on single-stack images and evaluated by measuring elongated fluorescent structures from the dendritic shaft to the beginning of the enlargement of the spine head. Dendritic protrusions showing a thin neck and finishing with no evident head 
enlargement were considered as filopodia. We matched the criteria used for spine identification in the experiment on fixed tissue and in-vivo.

Two-photon imaging. Pups born from 19 pregnant females were screened to obtain 20 males MeCP2-KO mice. From this population only 5 males MeCP2-KO provided usable imaging data, because of the high levels of mortality already described postweaning and because of adverse reaction to anesthesia and surgery ${ }^{12}$. All experiments have been performed on mice aged P25-P26. Animals were oxygenated, kept warm and respiratory and cardiac rhythms were monitored during both surgery and imaging. Since Rett syndrome is frequently associated with respiratory problems and ipocapnia ${ }^{49}$, we took extreme care in the control of the anesthesia levels. The study included only mice that maintained stationary breathing and cardiac rhythm and that did not display any other experimental anomalies like dendritic blebbing or excessive edema. Imaging experiments could not be performed blind because Rett mice were smaller in size than wild type mice as reported by 3 and 12 and furthermore anesthetic dosage had to be regulated differently in Rett mice. Indeed, we determined that RTT mice required a smaller dose of averthine than the control littermates: $0.04 \mathrm{ml} \times 10 \mathrm{~g}$ (RTT) versus $0.2 \mathrm{ml} \times 10 \mathrm{~g}$ (control). A cranial window was implanted at P25-26 according to a protocol described in 8 . Briefly, we opened a small craniotomy (about $4 \mathrm{~mm}$ of diameter) over the somatosensory cortex ( $2 \mathrm{~mm}$ posterior from bregma, $2 \mathrm{~mm}$ lateral from lambda) leaving the dura mater intact. The ECG was picked up by two tiny copper clips placed on the forelimbs. The signal was fed with shielded coaxial cables to a differential amplifier (NPI) and then to a custom made threshold discriminator that converted the heart beat waveform into a TTL signal used for imaging synchronization. Two-photon imaging was carried out on a dedicated 2-photon microscope (Ultima IV, Prairie Technology, Wisconsin) equipped with a $10 \mathrm{~W}$ laser (Coherent) tuned at $890 \mathrm{~nm}$ that delivered $30-50 \mathrm{~mW}$ at the sample. Imaging was restricted to the apical dendrites of layer $\mathrm{V}$ pyramidal neurons present in cortical layers II/III (50-150 $\mu \mathrm{m}$ below the cortical surface). Imaging was conducted for at least 50-60 min at an interval of 5 minutes and was interrupted if the mouse vital signs degraded. Images were acquired with a water immersion lens (Olympus, $60 \times$ NA 0.95 ) at a resolution of $512 \times 512$ pixels at zoom 4 , leading to a field of $50.7 \times 50.7 \mu \mathrm{m}$ and a nominal linear resolution of about $0.1 \mu \mathrm{m} /$ pixel. The stack step size was $0.75 \mu \mathrm{m}$. We selected the dendrites with the highest levels of GFP expression; moreover, we chose dendrites roughly parallel to the cortical surface to limit the depth of the z-stacks required to enclose the entire dendrite. The acquisition of each frame was synchronized to the hearth beat and this procedure strongly reduced the mechanical artifact produced by the pulsing brain circulation and improved the alignment of the image stacks.

Image analysis. Analysis of the imaging data was carried out using ImageJ. The optical sections of each stack were aligned to compensate for mechanical movements with a custom macro that used the RegStack plug in ${ }^{50}$. After alignment of the $\mathrm{z}$-stack obtained at each time point, the length of each spine was measured starting from the tip along the neck to the center of the dendrite. These measures were performed with a custom software that allowed to move easily along the z-stack to identify the spine features on the sections of optimum focus. For each spine we obtained a temporal series holding the spine length at each time. Usually the motility is described by computing the mean value of the derivative of the length. Since this measure scales with the mean length of the dendritic spine and in RTT mice spines are shorter, we decided to estimate the spine motility as the standard deviation of the series normalized to the mean spine length. The head volume has been computed at each temporal point by normalizing the integrated fluorescence (after background subtraction) from a ROI draw around the spine head with the mean fluorescence of the nearby dendrite. This normalization compensates for instrumental variations and also allows to compare measures made on different mice that have different levels of GFP expression ${ }^{51}$. The variability of the spine head has been quantified as the standard deviation of the volume normalized to the mean volume of each spine head. This analysis has been performed only on the spines characterized by a clearly defined head.

IGF-1 treatment. Subcutaneous injections of IGF-1 were performed by using IGF-1 (IU100; $1.8 \mu \mathrm{g} / \mathrm{g}$ body weight; Biovision $)^{52-55}$ in Rett null mice $(\mathrm{n}=9)$. We performed a single injection of this factor $24 \mathrm{~h}$ before imaging. According the concentration used in 22 , we injected $6 \mu \mathrm{l}$ of a solution made by $1 \mu \mathrm{l}$ of IGF-1 dissolved in saline. Subcutaneous injections were performed using a catheter with a 20 gauge needle connected to a Hamilton syringe. Imaging was successful in 6 RTT mice and 5 control mice.

Statistics. Statistical analysis was performed by Student t-test using Origin 7 software (OriginLab Co., Northampton, MA) and Sigma Stat 3.0 (SPSS Inc.). Cumulative frequencies of dendritic spine neck, mean spines length, motility and spine head fluctuations in KO and WT mice were compared using, first, the normal distribution Kolmogorov-Smirnov (KS) fitting test and then KS two-sample tests for subsequent paired comparisons (http://www.physics.csbsju.edu/stats/KS-test.html).

1. Chahrour, M. \& Zoghbi, H. Y. The story of Rett syndrome: from clinic to neurobiology. Neuron. 56, 422-37 (2007).

2. Bird, A. The methyl-CpG-binding protein MeCP2 and neurological disease. Biochem. Soc. Trans. 36, 575-83 (2008).
3. Zoghbi, H. Y. Postnatal neurodevelopmental disorders: meeting at the synapse? Science. 302, 826-830 (2003).

4. Boggio, E. M., Lonetti, G., Pizzorusso, T., Giustetto, M. Synaptic determinants of rett syndrome. Front. Syn. Neurosci. 2, 28 (2010).

5. Belichenko, P. V., Oldfors, A., Hagberg, B. \& Dahlström, A. Rett syndrome: 3-D confocal microscopy of cortical pyramidal dendrites and afferents. Neuroreport. 5, 1509-13 (1994).

6. Chapleau, C. A. et al Dendritic spine pathologies in hippocampal pyramidal neurons from Rett syndrome brain and after expression of Rett-associated MECP2 mutations. Neurobiol. Dis. 35, 219-33 (2009).

7. Belichenko, P. V.et al Widespread changes in dendritic and axonal morphology in Mecp2-mutant mouse models of Rett syndrome: evidence for disruption of neuronal networks. J. Comp. Neurol. 514, 240-258 (2009).

8. Holtmaat, A. et al Long-term, high-resolution imaging in the mouse neocortex through a chronic cranial window. Nat. Protoc. 4, 1128-44 (2009).

9. Chen, R. Z., Akbarian, S., Tudor, M. \& Jaenisch R. Deficiency of methyl-CpG binding protein-2 in CNS neurons results in a Rett-like phenotype in mice. Nat Genet 27, 327-31 (2001).

10. Feng, G. et al Imaging neuronal subsets in transgenic mice expressing multiple spectral variants of GFP. Neuron. 28, 41-51 (2000).

11. Stearns, N. A., Schaevitz, L. R., Bowling, H., Nag, N., Berger, U. V. \& BergerSweeney, J. Behavioral and anatomical abnormalities in Mecp2 mutant mice: a model for Rett syndrome. Neuroscience. 146, 907-21 (2007).

12. Lonetti, G. et al Early environmental enrichment moderates the behavioral and synaptic phenotype of MeCP2 null mice. Biol. Psychiatry. 67, 657-65 (2010).

13. Nishijima, T et al Neuronal activity drives localized blood-brain-barrier transport of serum insulin-like growth factor-I into the CNS. Neuron. 67, 834-46 (2010).

14. Riikonen, R. Neurotrophic factors in the pathogenesis of Rett syndrome. J. Child. Neurol. 18, 693-7 (2003).

15. Riikonen, R. Insulin-like growth factor delivery across the blood-brain barrier. Potential use of IGF-1 as a drug in child neurology. Chemotherapy. 52, 279-81 (2006).

16. Tropea, D. et al Partial reversal of Rett Syndrome-like symptoms in MeCP2 mutant mice. Proc. Natl. Acad.Sci. U S A. 106, 2029-34(2009).

17. Holtmaat, A. \& Svoboda, K. Experience-dependent structural synaptic plasticity in the mammalian brain. Nat. Rev. Neurosci. 10, 647-58 (2009).

18. Matsuzaki, M., Honkura, N., Ellis-Davies, G. C., Kasai, H. Structural basis of longterm potentiation in single dendritic spines. Nature. 429, 761-6 (2004).

19. Harvey, C. D. \& Svoboda, K. Locally dynamic synaptic learning rules in pyramidal neuron dendrites. Nature. 450, 1195-200 (2007).

20. Arellano, J. I., Benavides-Piccione, R., Defelipe, J. \& Yuste, R. Ultrastructure of dendritic spines: correlation between synaptic and spine morphologies. Front. Neurosci. 1, 131-43 (2007).

21. Nieto-Bona, M. P., Garcia-Segura, L. M. \& Torres-Alemán, I. Transynaptic modulation by insulin-like growth factor I of dendritic spines in Purkinje cells. Int. J. Dev. Neurosci. 15, 749-54 (1997).

22. Landi, S., Ciucci, F., Maffei, L., Berardi, N., Cenni, M. C. Setting the pace for retinal development: environmental enrichment acts through insulin-like growth factor 1 and brain-derived neurotrophic factor. J. Neurosci. 29, 10809-19 (2009).

23. D'Ercole, A. J., Ye, P., Calikoglu, A. S. \& Gutierrez-Ospina, G. The role of the insulin-like growth factors in the central nervous system. Mol. Neurobiol. 13, 22755 (1996).

24. O’Kusky, J. R., Ye, P. \& D’Ercole, A. J. Insulin-like growth factor-I promotes neurogenesis and synaptogenesis in the hippocampal dentate gyrus during postnatal development. J. Neurosci. 20, 8435-42 (2000).

25. Tropea, D. et al Gene expression changes and molecular pathways mediating activity-dependent plasticity in visual cortex. Nat. Neurosci. 9, 660-8 (2006).

26. Riikonen, R. et al Cerebrospinal fluid insulin-like growth factors IGF-1 and IGF-2 in infantile autism. Dev. Med. Child. Neurol. 48, 751-5 (2006).

27. Itoh, M. et al Methyl CpG-binding protein 2 (a mutation of which causes Rett syndrome) directly regulates insulin-like growth factor binding protein 3 in mouse and human brains. J. Neuropathol. Exp. Neurol. 66, 117-23 (2007).

28. Thoenen, H. \& Sendtner, M. Neurotrophins: from enthusiastic expectations through sobering experiences to rational therapeutic approaches. Nat Neurosci. 5, 1046-50 (2002).

29. Baker, A. M., Batchelor, D. C., Thomas, G. B.,Wen, J. Y., Rafiee,M., Lin, H., \& Guan, J. Central penetration and stability of N-terminal tripeptide of insulin-like growth factor-I, glycine-proline-glutamate in adult rat. Neuropeptides 39, 81-87 (2005).

30. Pardridge, W. M. Transport of insulin-related peptides and glucose across the blood-brain barrier. Ann. N Y Acad. Sci. 692, 126-137 (1993).

31. Reinhardt, R. R., \& Bondy, C. A. Insulin-like growth factors cross the blood-brain barrier. Endocrinology 135, 1753-1761 (1994).

32. Carro, E., Nunez, A., Busiguina, S., \& Torres-Aleman, I. Circulating insulin-like growth factor I mediates effects of exercise on the brain. J. Neurosci. 20, 29262933 (2000).

33. Pulford, B. E., \& Ishii, D. N. Uptake of circulating insulin-like growth factors (IGFs) into cerebrospinal fluid appears to be independent of the IGF receptors as well as IGF-binding proteins. Endocrinology 142, 213-220 (2001).

34. Trejo, J. L., Carro, E. \& Torres-Aleman, I. Circulating insulin-like growth factor I mediates exercise-induced increases in the number of new neurons in the adult hippocampus. J Neurosci 21, 1628-1634 (2001). 
35. Garcia-Segura, L. M., Perez, J., Pons, S., Rejas, M. T., \& Torres-Aleman, I. Localization of insulin-like growth factor I (IGF-I)-like immunoreactivity in the developing and adult rat brain. Brain Res. 560, 167-174 (1991).

36. Marks, J. L., Porte, D., Jr., \& Baskin, D. G. Localization of type I insulin-like growth factor receptor messenger RNA in the adult rat brain by in situ hybridization. Mol. Endocrinol. 5, 1158-1168 (1991).

37. Auletta, M., Nielsen, F. C., \& Gammeltoft, S. Receptor-mediated endocytosis and degradation of insulin-like growth factor I and II in neonatal rat astrocytes. J. Neurosci. Res. 31, 14-20 (1992)

38. Yoshii, A. \& Constantine-Paton, M. BDNF induces transport of PSD-95 to dendrites through PI3K-AKT signaling after NMDA receptor activation. Nat. Neurosci. 10, 702-11 (2007).

39. Carvalho, A. L., Caldeira, M. V., Santos, S. D. \& Duarte, C. B. Role of the brainderived neurotrophic factor at glutamatergic synapses. Br J Pharmacol 153, S31024 (2008).

40. Ricciardi, S. et al Reduced AKT/mTOR signaling and protein synthesis dysregulation in a Rett syndrome animal model. Hum. Mol. Genet. 20, 1182-96 (2011)

41. Wang, H. et al Dysregulation of brain-derived neurotrophic factor expression and neurosecretory function in Mecp2 null mice. J. Neurosci. 26, 10911-5 (2006)

42. Chang, Q., Khare, G., Dani, V., Nelson, S. \& Jaenisch, R. The disease progression of Mecp2 mutant mice is affected by the level of BDNF expression. Neuron. 49, 3418 (2006)

43. Ramsey, M. M., Adams, M. M., Ariwodola, O. J., Sonntag, W. E. \& Weiner, J. L. Functional characterization of des-IGF-1 action at excitatory synapses in the CA1 region of rat hippocampus. J. Neurophysiol. 94, 247-54 (2005).

44. Marchetto, M. C. et al A model for neural development and treatment of Rett syndrome using human induced pluripotent stem cells. Cell. 143, 527-39 (2010).

45. Takano, K. et al Nebulin and N-WASP cooperate to cause IGF-1-induced sarcomeric actin filament formation. Science. 330, 1536-40 (2010).

46. Wegner, A. M. et al $\mathrm{N}$-wasp and the arp $2 / 3$ complex are critical regulators of actin in the development of dendritic spines and synapses. J. Biol. Chem. 283, 15912-20 (2008).

47. Wolfer, D. P., Crusio, W. E. \& Lipp, H. P. Knockout mice: simple solutions to the problems of genetic background and flanking genes. Trends. Neurosci. 25, 336340(2002).

48. Pavlowsky, A., et al A postsynaptic signaling pathway that may account for the cognitive defect due to IL1RAPL1 mutation. Curr. Biol. 20, 103-15 (2010).

49. Voituron, N. et al Early abnormalities of post-sigh breathing in a mouse model of Rett syndrome. Respir. Physiol. Neurobiol. 170, 173-82 (2010).

50. Thévenaz, P., Ruttimann, U. E., Unser, M. A pyramid approach to subpixel registration based on intensity. IEEE Transactions on Image Processing vol. 7, no. 1, pp. 27-41 (1998).
51. Cruz-Martín, A., Crespo, M. \& Portera-Cailliau, C. Delayed stabilization of dendritic spines in fragile X mice. J. Neurosci. 30, 7793-803 (2010).

52. Humbel, R. E. Insulin-like growth factors I and II. Eur. J. Biochem. 190, 445-462 (1990).

53. Stewart, C. E. \& Rotwein, P. Growth, differentiation, and survival: multiple physiological functions for insulin-like growth factors. Physiol. Rev. 7, 1005-1026 (1996).

54. Woodall, S. M. et al Administration of growth hormone or IGF-I to pregnant rats on a reduced diet throughout pregnancy does not prevent fetal intrauterine growth retardation and elevated blood pressure in adult offspring. J. Endocrinol. 163, 69-77 (1999).

55. Thongsong, B. et al Effects of insulin-like growth factor-I on maternal and foetal plasma amino acid levels in pregnant rats. J. Vet. Med. Sci. 64, 859-861 (2002).

\section{Acknowledgements}

We thank Paolo Orsini for custom software for spine motility analysis. Thanks for supporting this project to the following grants: FIRB 2003 Laboratorio nazionale sulle nanotecnologie per genomica e post-genomica (NG-Lab), Telethon Grant GGP07278 to G.M.R. and GGP09196 to T.P. and M.G., TLS-Toscana Life Sciences 'Orphan diseases' to G.M.R., Firb FUTURO IN RICERCA2008-Miur to S.L. and the E-Rare EuroRett Consortium to T.P. and M.G. We acknowledge the help of other members of the lab, specifically of Marco Brondi, Sebastian Sulis Sato, Luisa de Vivo and Mariangela Panniello.

\section{Author contributions}

S.L., E.P., T.P., G.M.R. designed the study. E.M.B. and M.G. performed the experiments on fixed tissue. The in vivo imaging experiments have been performed by S.L. and E.P. under the supervision of G.M.R. S.L. and G.M.R. prepared the manuscript and all authors contributed to the final form.

\section{Additional information}

Supplementary Information accompanies this paper at http://www.nature.com/ scientificreports

Competing financial interests: Authors have no Competing Financial Interests.

License: This work is licensed under a Creative Commons

Attribution-NonCommercial-ShareAlike 3.0 Unported License. To view a copy of this license, visit http://creativecommons.org/licenses/by-nc-sa/3.0/

How to cite this article: Landi, S. et al. The short-time structural plasticity of dendritic spines is altered in a model of Rett syndrome. Sci. Rep. 1, 45; DOI:10.1038/srep00045 (2011) 